\title{
Erratum to: On the set-semidefinite representation of nonconvex quadratic programs over arbitrary feasible sets
}

\author{
Peter J. C. Dickinson • Gabriele Eichfelder • \\ Janez Povh
}

Published online: 3 July 2013

(C) Springer-Verlag Berlin Heidelberg 2013

\section{Erratum to: Optim Lett DOI 10.1007/s11590-012-0450-3}

In this paper, an erratum is provided to the article "On the set-semidefinite representation of nonconvex quadratic programs over arbitrary feasible sets", published in Optim Lett, 2012. Due to precise observation of the first author, it has been found that the proof of Lemma 9 has a nontrivial gap, and consequently the main result (Theorem 10) is incorrect. In this erratum, we prove that Corollary 14 is still correct in the original setting while to fix the proof of Theorem 10 we need additional assumptions. We provide a list of different commonly used assumptions making this theorem to be true, and a new version of this theorem, which is now Theorem 17.

The online version of the original article can be found under doi:10.1007/s11590-012-0450-3.

\section{P. J. C. Dickinson}

Department of Statistics and Operations Research, University of Vienna,

Oskar-Morgenstern-Platz 1, 1090 Wien, Austria

e-mail: peter.dickinson@univie.ac.at

G. Eichfelder $(\bowtie)$

Institute of Mathematics, Ilmenau University of Technology, PO 1005 65,

98684 Ilmenau, Germany

e-mail: Gabriele.Eichfelder@tu-ilmenau.de

\section{J. Povh}

Faculty of information studies in Novo mesto, Ulica talcev 3, 8000 Novo mesto, Slovenia

e-mail: janez.povh@fis.unm.si 


\section{The error}

The second and the third author of this paper considered, in [4], the following quadratic optimization problem as an extension of that considered in [3], where $\mathcal{K} \subseteq \mathbb{R}^{n}$ is a nonempty set, $\mathcal{B} \subseteq\{1, \ldots, n\}$ is an index set, $Q \in \mathcal{S}^{n}$ is a symmetric matrix, $\mathbf{b} \in \mathbb{R}^{m}$ and $\mathbf{c} \in \mathbb{R}^{n}$ are vectors, and $A \in \mathbb{R}^{m \times n}$ is a matrix, with $\mathbf{a}_{1}^{\top}, \ldots, \mathbf{a}_{m}^{\top} \in \mathbb{R}^{n}$ denoting its rows:

$$
\begin{array}{lll}
\inf _{\mathbf{x} \in \mathbb{R}^{n}} & \mathbf{x}^{\top} Q \mathbf{x}+2 \mathbf{c}^{\top} \mathbf{x} & \\
\text { s.t. } & A \mathbf{x}=\mathbf{b} & \\
& x_{j} \in\{0,1\} \quad \text { for all } j \in \mathcal{B} \\
& \mathbf{x} \in \mathcal{K} . &
\end{array}
$$

An assumption connected with this problem is as follows:

Assumption 1 If we have $\mathbf{x} \in \mathcal{K}$ such that $A \mathbf{x}=b$ then $x_{j} \in[0,1]$ for all $j \in B$.

This assumption can be made to hold by either adding the constraints directly to $\mathcal{K}$ or by adding slack variables with nonnegative constraints.

It was claimed that if Assumption 1 holds then $\left(\mathrm{QP}_{\mathrm{C}}\right)$, as given below, is a reformulation of (QP), by which we mean that the optimal values of both problems are equal.

$$
\begin{array}{cl}
\inf _{(\mathbf{x}, X) \in \mathbb{R}^{n} \times \mathcal{S}^{n}} & \langle Q, X\rangle+2 \mathbf{c}^{\top} \mathbf{x} \\
\text { s.t. } & A \mathbf{x}=\mathbf{b} \\
& \operatorname{Diag}\left(A X A^{\top}\right)=\mathbf{b} \circ \mathbf{b} \quad \\
& x_{j}=X_{j j} \quad \text { for all } j \in \mathcal{B} \\
& \left(\begin{array}{cc}
1 & \mathbf{x}^{\top} \\
\mathbf{x} & X
\end{array}\right) \in \mathcal{C}_{\{1\} \times \mathcal{K},}^{*},
\end{array}
$$

where $(\mathbf{b} \circ \mathbf{b})^{\top}:=\left(b_{1}^{2}, b_{2}^{2}, \ldots, b_{m}^{2}\right)^{\top}$, i.e. the Hadamard product. Also recall from [4, Lemma 4] that for a set $\mathcal{L} \subseteq \mathbb{R}^{n+1}$ we have

$$
\begin{aligned}
\mathcal{C}_{\mathcal{L}}^{*} & =\operatorname{cl} \text { conv cone }\left\{\mathbf{x x}^{\top} \mid \mathbf{x} \in \mathcal{L}\right\} \\
& =\operatorname{conv}\left\{\mathbf{x} \mathbf{x}^{\top} \mid \mathbf{x} \in \operatorname{cl} \text { cone } \mathcal{L}\right\} .
\end{aligned}
$$

The first author has constructed the following two counter examples showing that Assumption 1 holding is not a sufficient condition for $\left(\mathrm{QP}_{\mathrm{C}}\right)$ being a reformulation of (QP):

Example 1 Let us consider the following example for (QP) with $n=1, m=0$, $\mathcal{B}=\{1\}$ and $\mathcal{K}=(0,1]$ :

$$
\inf \{x \in \mathbb{R} \mid x \in\{0,1\}, \quad x \in \mathcal{K}\}=1 .
$$


Assumption 1 holds for the problem, and the corresponding version of $\left(\mathrm{QP}_{\mathrm{C}}\right)$ is

$$
\inf \left\{x \in \mathbb{R} \mid\left(\begin{array}{ll}
1 & x \\
x & x
\end{array}\right) \in \mathcal{C}_{\{1\} \times \mathcal{K}}^{*}\right\}
$$

We have that $\left(\begin{array}{l}1 \\ x\end{array}\right)\left(\begin{array}{l}1 \\ x\end{array}\right)^{\top} \in \mathcal{C}_{\{1\} \times \mathcal{K}}^{*}$ for all $x \in(0,1]$, therefore, as $\mathcal{C}_{\{1\} \times \mathcal{K}}^{*}$ is closed,

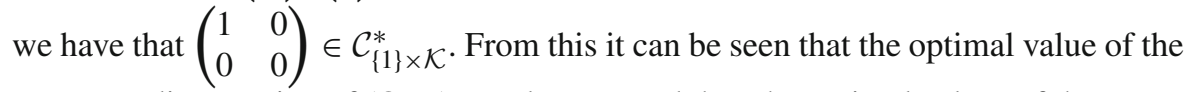
corresponding version of $\left(\mathrm{QP}_{\mathrm{C}}\right)$ equals zero, and thus the optimal values of these two problems are not equal.

Example 2 We let

$$
\begin{aligned}
\mathcal{K}_{1} & =\text { conv cone }\left\{\left(\begin{array}{l}
1 \\
0 \\
1
\end{array}\right),\left(\begin{array}{l}
1 \\
1 \\
1
\end{array}\right),\left(\begin{array}{l}
1 \\
0 \\
0
\end{array}\right)\right\} \\
& =\left\{\mathbf{x} \in \mathbb{R}^{3} \mid x_{1}-x_{3} \geq 0, \quad x_{3}-x_{2} \geq 0, \quad x_{2} \geq 0\right\} \\
\mathcal{K}_{2} & =\text { conv cone }\left\{\left(\begin{array}{l}
1 \\
0 \\
1
\end{array}\right),\left(\begin{array}{l}
1 \\
1 \\
1
\end{array}\right),\left(\begin{array}{l}
1 \\
1 \\
0
\end{array}\right)\right\} \\
& =\left\{\mathbf{x} \in \mathbb{R}^{3} \mid x_{1}-x_{3} \geq 0, \quad x_{1}-x_{2} \geq 0, \quad-x_{1}+x_{2}+x_{3} \geq 0\right\}
\end{aligned}
$$

We now consider the following example for (QP) with $n=3, m=2, \mathcal{B}=\emptyset$ and $\mathcal{K}=\mathcal{K}_{1} \cup \mathcal{K}_{2}$ :

$$
\inf \left\{-x_{1}^{2} \mid x_{2}=3, \quad x_{3}=2, \quad \mathbf{x} \in \mathcal{K}\right\}
$$

It can be seen that

$$
\begin{aligned}
& \left\{\mathbf{x} \in \mathcal{K}_{1} \mid x_{2}=3, \quad x_{3}=2\right\}=\emptyset \\
& \left\{\mathbf{x} \in \mathcal{K}_{2} \mid x_{2}=3, \quad x_{3}=2\right\}=\operatorname{conv}\left\{\left(\begin{array}{l}
3 \\
3 \\
2
\end{array}\right),\left(\begin{array}{l}
5 \\
3 \\
2
\end{array}\right)\right\}
\end{aligned}
$$

Therefore the optimal value for the problem $(\mathrm{QP})$ is equal to -25 . One can however verify that the corresponding version of $\left(\mathrm{QP}_{\mathrm{C}}\right)$ has the optimal value $-\infty$ and thus the optimal values of these two problems are not equal.

The error in the paper in fact comes from [4, Lemma 9], in the last paragraph of the proof. In this, the mistake was effectively in assuming that for a set $\mathcal{Y}_{1}$ defined by linear equality constraints and a convex set $\mathcal{Y}_{2}$, we have that $\mathcal{Y}_{1} \cap\left(\operatorname{cl} \mathcal{Y}_{2}\right) \subseteq \operatorname{cl}\left(\mathcal{Y}_{1} \cap \mathcal{Y}_{2}\right)$, which is not true in general. 


\section{Repairing the error}

In this section we essentially provide two new versions of Theorem 10 from [4], where we decided to provide a detailed study of two different situations where Theorem 10 still holds. We do this using the asymptotic cone, where we recall that for a nonempty set $\mathcal{K} \subseteq \mathbb{R}^{n}$, the asymptotic cone is defined as follows and we note that this is always a closed cone:

$\mathcal{K}_{\infty}:=\left\{\mathbf{x} \in \mathbb{R}^{n} \mid \exists\left\{\left(\lambda_{i}, \mathbf{x}_{i}\right) \mid i \in \mathbb{N}\right\} \subseteq \mathbb{R}_{+} \times \mathcal{K} \quad\right.$ such that $\left.\quad \lim _{i \rightarrow \infty} \lambda_{i}=0, \quad \lim _{i \rightarrow \infty} \lambda_{i} \mathbf{x}_{i}=\mathbf{x}\right\}$

We begin by presenting the following lemma from [1], where they used an equivalent definition of the asymptotic cone.

Lemma 3 [1, Lemma 2.1.1] Let $\mathcal{K} \subseteq \mathbb{R}^{n}$ be a nonempty closed set. Then

$$
\mathrm{cl} \operatorname{cone}(\{1\} \times \mathcal{K})=\operatorname{cone}(\{1\} \times \mathcal{K}) \cup\left(\{0\} \times \mathcal{K}_{\infty}\right) .
$$

Together with Lemma 4 in [4] we obtain the following.

Corollary 4 Let $\mathcal{K} \subseteq \mathbb{R}^{n}$ be a nonempty closed set. Then

$$
\mathcal{C}_{\{1\} \times \mathcal{K}}^{*}=\operatorname{conv}\left\{\mathbf{z z}^{\top} \mid \mathbf{z} \in \operatorname{cone}(\{1\} \times \mathcal{K}) \cup\left(\{0\} \times \mathcal{K}_{\infty}\right)\right\} .
$$

By applying Carathéodory's Theorem we get the following corollary.

Corollary 5 Let $\mathcal{K} \subseteq \mathbb{R}^{n}$ be a nonempty closed set, let $Y \in \mathcal{C}_{\{1\} \times \mathcal{K}}^{*}$ and let $M$ equal the dimension of the space containing $\mathcal{C}_{\{1\} \times \mathcal{K}}^{*}$. Then there exists $\lambda_{1}, \ldots, \lambda_{p}>0$ and $\mathbf{x}_{1}, \ldots, \mathbf{x}_{p} \in \mathcal{K}$ and $\mathbf{d}_{1}, \ldots, \mathbf{d}_{q} \in \mathcal{K}_{\infty}$ such that $p+q=M$ and

$$
Y=\sum_{i=1}^{p} \lambda_{i}\left(\begin{array}{c}
1 \\
\mathbf{x}_{i}
\end{array}\right)\left(\begin{array}{c}
1 \\
\mathbf{x}_{i}
\end{array}\right)^{\top}+\sum_{i=1}^{q}\left(\begin{array}{c}
0 \\
\mathbf{d}_{i}
\end{array}\right)\left(\begin{array}{c}
0 \\
\mathbf{d}_{i}
\end{array}\right)^{\top} .
$$

We define the following sets related to the problems $(\mathrm{QP})$ and $\left(\mathrm{QP}_{\mathrm{C}}\right)$

$$
\begin{aligned}
\text { Feas }^{+}(\mathrm{QP}): & =\operatorname{conv}\left\{\left(\begin{array}{l}
1 \\
\mathbf{x}
\end{array}\right)\left(\begin{array}{l}
1 \\
\mathbf{x}
\end{array}\right)^{\top} \mid \mathbf{x} \in \operatorname{Feas}(\mathrm{QP})\right\}, \\
\text { Feas }^{+}\left(\mathrm{QP}_{\mathrm{C}}\right): & =\left\{\left(\begin{array}{cc}
1 & \mathbf{x}^{\top} \\
\mathbf{x} & X
\end{array}\right) \mid(\mathbf{x}, X) \in \operatorname{Feas}\left(\mathrm{QP}_{\mathrm{C}}\right)\right\}, \\
L_{\infty}: & =\left\{\mathbf{d} \in \mathcal{K}_{\infty} \mid \mathbf{a}_{j}^{\top} \mathbf{d}=0 \text { for all } j=1, \ldots, m\right\}, \\
L_{\infty}^{+}: & =\operatorname{conv}\left\{\left(\begin{array}{l}
0 \\
\mathbf{d}
\end{array}\right)\left(\begin{array}{l}
0 \\
\mathbf{d}
\end{array}\right)^{\top} \mid \mathbf{d} \in L_{\infty}\right\} .
\end{aligned}
$$

Note that this notation is the same as that used in [2-4] with one difference that the definition of $L_{\infty}$ uses the asymptotic cone. 
We now define the following assumptions. Although these assumptions are currently quite technical, later we shall show that some combinations of them can be implied by simpler assumptions.

Assumption $2 \mathcal{K} \subseteq \mathbb{R}^{n}$ is closed, and, if there exists an $\mathbf{x} \in \mathcal{K}$ such that $A \mathbf{x}=\mathbf{b}$, then $(\mathbf{d})_{j}=0$ for all $\mathbf{d} \in L_{\infty}, j \in \mathcal{B}$.

Assumption 3 The optimal value of $\left(\mathrm{QP}_{\mathrm{C}}\right)$, denoted $\mathrm{Val}\left(\mathrm{QP}_{\mathrm{C}}\right)$, is not equal to $-\infty$.

Assumption 4 For all $\mathbf{d} \in L_{\infty}, \mathbf{y} \in \operatorname{Feas}(\mathrm{QP}), \underset{\widetilde{\lambda}}{\tilde{\lambda}} \in \mathbb{R}_{+}$, there $\underset{\widetilde{\lambda}]}{\operatorname{exists}} \underset{\widetilde{\lambda}}{\lambda} \in \mathbb{R} \backslash(-\tilde{\lambda}, \tilde{\lambda})$, such that $\mathbf{y}+\lambda \mathbf{d} \in \operatorname{Feas}(\mathrm{QP})$, where $\mathbb{R} \backslash(-\tilde{\lambda}, \widetilde{\lambda})=(-\infty,-\widetilde{\lambda}] \cup[\widetilde{\lambda}, \infty)$.

We now present the following results connected to these assumptions. In these, we consider the Minkowski sum, i.e. for two sets $\mathcal{X}, \mathcal{Y}$, we define $\mathcal{X}+\mathcal{Y}:=\{\mathbf{x}+\mathbf{y} \mid \mathbf{x} \in \mathcal{X}, \mathbf{y} \in \mathcal{Y}\}$, and we note that $\emptyset+\mathcal{X}=\emptyset$.

Lemma 6 If Assumption 2 holds then Feas $^{+}(\mathrm{QP})+L_{\infty}^{+} \subseteq$ Feas $^{+}\left(\mathrm{QP}_{\mathrm{C}}\right)$.

Proof This follows immediately from Corollary 4.

Lemma 7 Consider problems $(\mathrm{QP})$ and $\left(\mathrm{QP}_{\mathrm{C}}\right)$ such that Assumptions 1 and 2 hold. Then Feas ${ }^{+}\left(\mathrm{QP}_{\mathrm{C}}\right)=$ Feas $^{+}(\mathrm{QP})+\mathrm{L}_{\infty}^{+}$.

Proof From Lemma 6 we have Feas ${ }^{+}(\mathrm{QP})+L_{\infty}^{+} \subseteq$ Feas $^{+}\left(\mathrm{QP}_{\mathrm{C}}\right)$.

We shall now show that Feas ${ }^{+}(\mathrm{QP})+L_{\infty}^{+} \supseteq$ Feas $^{+}\left(\mathrm{QP}_{\mathrm{C}}\right)$. If Feas ${ }^{+}\left(\mathrm{QP}_{\mathrm{C}}\right)=\emptyset$ then $\emptyset=$ Feas $^{+}(\mathrm{QP})+L_{\infty}^{+}$and we are done. From now on we assume that Feas $^{+}\left(\mathrm{QP}_{\mathrm{C}}\right) \neq \varnothing$ and consider an arbitrary $\left(\begin{array}{cc}1 & \mathbf{x}^{\top} \\ \mathbf{x} & X\end{array}\right) \in$ Feas $^{+}\left(\mathrm{QP}_{\mathrm{C}}\right)$, which we will show to be in Feas ${ }^{+}(\mathrm{QP})+L_{\infty}^{+}$.

From Corollary 5 , there exists $\lambda_{1}, \ldots, \lambda_{p}>0$ and $\mathbf{x}_{1}, \ldots, \mathbf{x}_{p} \in \mathcal{K}$ and $\mathbf{d}_{1}, \ldots, \mathbf{d}_{q} \in \mathcal{K}_{\infty}$ with $p+q=M$, where $M$ equals the dimension of the space containing $\mathcal{C}_{\{1\} \times \mathcal{K}}^{*}$, such that

$$
\left(\begin{array}{cc}
1 & \mathbf{x}^{\top} \\
\mathbf{x} & X
\end{array}\right)=\sum_{i=1}^{p} \lambda_{i}\left(\begin{array}{c}
1 \\
\mathbf{x}_{i}
\end{array}\right)\left(\begin{array}{c}
1 \\
\mathbf{x}_{i}
\end{array}\right)^{\top}+\sum_{i=1}^{q}\left(\begin{array}{c}
0 \\
\mathbf{d}_{i}
\end{array}\right)\left(\begin{array}{c}
0 \\
\mathbf{d}_{i}
\end{array}\right)^{\top}
$$

We have $1=\sum_{i=1}^{p} \lambda_{i}$ and thus $p>0$. For all $j=1, \ldots, m$ we have

$$
\begin{aligned}
0 & =b_{j}^{2}-2 b_{j}^{2}+b_{j}^{2} \\
& =\mathbf{a}_{j}^{\top} X \mathbf{a}_{j}-2 b_{j}\left(\mathbf{a}_{j}^{\top} \mathbf{x}\right)+b_{j}^{2} \\
& =\sum_{i=1}^{p} \lambda_{i}\left(\mathbf{a}_{j}^{\top} \mathbf{x}_{i}\right)^{2}+\sum_{i=1}^{q}\left(\mathbf{a}_{j}^{\top} \mathbf{d}_{i}\right)^{2}-2 b_{j} \sum_{i=1}^{p} \lambda_{i}\left(\mathbf{a}_{j}^{\top} \mathbf{x}_{i}\right)+b_{j}^{2} \sum_{i=1}^{p} \lambda_{i} \\
& =\sum_{i=1}^{q} \underbrace{\left(\mathbf{a}_{j}^{\top} \mathbf{d}_{i}\right)^{2}}_{\geq 0}+\sum_{i=1}^{p} \underbrace{\lambda_{i}}_{>0} \underbrace{\left(\mathbf{a}_{j}^{\top} \mathbf{x}_{i}-b_{j}\right)^{2}}_{\geq 0} \geq 0 .
\end{aligned}
$$


Therefore $\mathbf{d}_{i} \in L_{\infty}$ for all $i=1, \ldots, q$, and $\mathbf{a}_{j}^{\top} \mathbf{x}_{i}=b_{j}$ for all $i=1, \ldots, p$, $j=1, \ldots, m$. From Assumption 1, we have that $\left(\mathbf{x}_{i}\right)_{j} \in[0,1]$ for all $i=1, \ldots, p$, $j \in \mathcal{B}$, and from Assumption 2, we have that $\left(\mathbf{d}_{i}\right)_{j}=0$ for all $i=1, \ldots, q, j \in \mathcal{B}$. Using this, for all $j \in \mathcal{B}$ we have

$$
\begin{aligned}
0 & =(\mathbf{x})_{j}-(X)_{j j} \\
& =\sum_{i=1}^{p} \lambda_{i}\left(\mathbf{x}_{i}\right)_{j}-\sum_{i=1}^{p} \lambda_{i}\left(\mathbf{x}_{i}\right)_{j}^{2}-\sum_{i=1}^{q}\left(\mathbf{d}_{i}\right)_{j}^{2} \\
& =\sum_{i=1}^{p} \underbrace{\lambda_{i}}_{>0} \underbrace{\left(\mathbf{x}_{i}\right)_{j}\left(1-\left(\mathbf{x}_{i}\right)_{j}\right)}_{\geq 0} \geq 0 .
\end{aligned}
$$

Therefore $\left(\mathbf{x}_{i}\right)_{j} \in\{0,1\}$ for all $i=1, \ldots, p, j \in \mathcal{B}$, which implies that $\mathbf{x}_{i} \in \operatorname{Feas}(\mathrm{QP})$ for all $i=1, \ldots, p$. This along with our earlier observation that $\mathbf{d}_{i} \in L_{\infty}$ for all $i=1, \ldots, q$ completes the proof.

We now consider Assumption 3 in addition to Assumptions 1 and 2.

Theorem 8 Consider problems $(\mathrm{QP})$ and $\left(\mathrm{QP}_{\mathrm{C}}\right)$ such that Assumptions 1, 2 and 3 hold. Then $\operatorname{Val}(\mathrm{QP})=\operatorname{Val}\left(\mathrm{QP}_{\mathrm{C}}\right)$. Furthermore, if we let $\mathrm{Opt}(\mathrm{QP})$ and $\mathrm{Opt}\left(\mathrm{QP}_{\mathrm{C}}\right)$ be the set of optimal solutions of $(\mathrm{QP})$ and $\left(\mathrm{QP}_{\mathrm{C}}\right)$ respectively, then

$$
\begin{aligned}
\mathrm{Opt}^{+}\left(\mathrm{QP}_{\mathrm{C}}\right): & =\left\{\left(\begin{array}{cc}
1 & \mathbf{x}^{\top} \\
\mathbf{x} & X
\end{array}\right) \mid(\mathbf{x}, X) \in \mathrm{Opt}\left(\mathrm{QP}_{\mathrm{C}}\right)\right\} \\
& =\operatorname{conv}\left\{\left(\begin{array}{l}
1 \\
\mathbf{x}
\end{array}\right)\left(\begin{array}{l}
1 \\
\mathbf{x}
\end{array}\right)^{\top} \mid \mathbf{x} \in \mathrm{Opt}(\mathrm{QP})\right\}+\operatorname{conv}\left\{\left(\begin{array}{l}
0 \\
\mathbf{d}
\end{array}\right)\left(\begin{array}{l}
0 \\
\mathbf{d}
\end{array}\right)^{\top} \mid \begin{array}{l}
\mathbf{d} \in L_{\infty}, \\
\mathbf{d}^{\top} Q \mathbf{d}=0
\end{array}\right\}
\end{aligned}
$$

Proof From the previous lemma we have that Feas ${ }^{+}\left(\mathrm{QP}_{\mathrm{C}}\right)=\mathrm{Feas}^{+}(\mathrm{QP})+L_{\infty}^{+}$. We now split this proof into two cases:

Case 1 Feas $^{+}(\mathrm{QP})=\emptyset$ : Then Feas ${ }^{+}\left(\mathrm{QP}_{\mathrm{C}}\right)=\emptyset$ and the results immediately follow. Case 2 Feas $^{+}(\mathrm{QP}) \neq \emptyset$ : We shall show that $\langle Q, D\rangle \geq 0$ for all $\left(\begin{array}{ll}0 & \mathbf{0}^{\top} \\ \mathbf{0} & D\end{array}\right) \in L_{\infty}^{+}$, from which the required results immediately follow.

We consider an arbitrary $Z \in \mathrm{Feas}^{+}(\mathrm{QP})$ and $\left(\begin{array}{cc}0 & \mathbf{0}^{\top} \\ \mathbf{0} & D\end{array}\right) \in L_{\infty}^{+}$. As $L_{\infty}^{+}$is a cone, for all $\lambda \geq 0$ we have that $Z+\lambda\left(\begin{array}{cc}0 & \mathbf{0}^{\top} \\ \mathbf{0} & D\end{array}\right) \in \mathrm{Feas}^{+}(\mathrm{QP})+L_{\infty}^{+}=\mathrm{Feas}^{+}\left(\mathrm{QP}_{\mathrm{C}}\right)$. Therefore we must have that $\langle Q, D\rangle \geq 0$, otherwise $\operatorname{Val}\left(\mathrm{QP}_{\mathrm{C}}\right)=-\infty$.

We next consider Assumption 4 in addition to Assumptions 1 and 2.

Lemma 9 Consider problems $(\mathrm{QP})$ and $\left(\mathrm{QP}_{\mathrm{C}}\right)$ such that Assumptions 1, 2 and 4 hold. Then Feas $^{+}(\mathrm{QP})+L_{\infty}^{+}=$Feas $^{+}\left(\mathrm{QP}_{\mathrm{C}}\right)=\mathrm{cl}\left(\mathrm{Feas}^{+}(\mathrm{QP})\right)$.

Proof If Feas $(\mathrm{QP})=\emptyset$ then both Feas ${ }^{+}(\mathrm{QP})$ and Feas ${ }^{+}\left(\mathrm{QP}_{\mathrm{C}}\right)$ are empty. Therefore, in this case, $\emptyset=$ Feas $^{+}(\mathrm{QP})+L_{\infty}^{+}=$Feas $^{+}\left(\mathrm{QP}_{\mathrm{C}}\right)=\operatorname{cl}\left(\mathrm{Feas}^{+}(\mathrm{QP})\right)$, and we are done. From now on we shall assume that Feas $(\mathrm{QP}) \neq \emptyset$. 
From Lemma 7 we have Feas $^{+}(\mathrm{QP})+L_{\infty}^{+}=\mathrm{Feas}^{+}\left(\mathrm{QP}_{\mathrm{C}}\right)$, and in the remainder of this proof we shall show that we also have Feas $^{+}(\mathrm{QP})+L_{\infty}^{+} \subseteq \operatorname{cl}\left(\right.$ Feas $\left.^{+}(\mathrm{QP})\right) \subseteq \mathrm{Feas}^{+}\left(\mathrm{QP}_{\mathrm{C}}\right)$.

Feas ${ }^{+}\left(\mathrm{QP}_{\mathrm{C}}\right)$ is the intersection of closed sets, and so is itself closed. Therefore, we

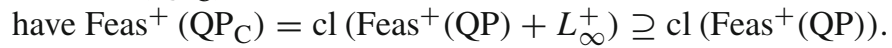

We now consider an arbitrary $Y \in$ Feas $^{+}(\mathrm{QP})$ and $\left(\begin{array}{cc}0 & \mathbf{0}^{\top} \\ \mathbf{0} & D\end{array}\right) \in L_{\infty}^{+}$. By Carathéodory's Theorem we have that for $M$ equal to the dimension of the space containing $\mathcal{C}_{\{1\} \times \mathcal{K}}^{*}$, there exists $\mathbf{d}_{1}, \ldots, \mathbf{d}_{M} \in L_{\infty}$ such that $D=\sum_{i=1}^{M} \mathbf{d}_{i} \mathbf{d}_{i}^{\top}$. We now consider an arbitrary $\mathbf{x} \in \mathrm{Feas}(\mathrm{QP})$ and note from Assumption 4, that there exists a set $\left\{\lambda_{i, j} \mid j \in \mathbb{N}, i=1, \ldots, M\right\}$ such that for all $i, j$ we have $\lambda_{i, j}^{2} \geq M \exp (j)$ and $\mathbf{x}+\lambda_{i, j} \mathbf{d}_{i} \in \operatorname{Feas}(\mathrm{QP})$. We then have the following for all $i, j$ :

$$
0 \leq \lambda_{i, j}^{-2}, \quad \sum_{i=1}^{M} \lambda_{i, j}^{-2} \leq \exp (-j) \leq 1
$$

From the definition of Feas $^{+}(\mathrm{QP})$ we have $\left(\begin{array}{c}1 \\ \mathbf{x}+\lambda_{i, j} \mathbf{d}_{i}\end{array}\right)\left(\begin{array}{c}1 \\ \mathbf{x}+\lambda_{i, j} \mathbf{d}_{i}\end{array}\right)^{\top} \in$ Feas $^{+}(\mathrm{QP})$ for all $i, j$. Now, using the fact that Feas $^{+}(\mathrm{QP})$ is convex, we have the following for all $j \in \mathbb{N}$ :

$$
\left[\sum_{i=1}^{M} \lambda_{i, j}^{-2}\left(\begin{array}{c}
1 \\
\mathbf{x}+\lambda_{i, j} \mathbf{d}_{i}
\end{array}\right)\left(\begin{array}{c}
1 \\
\mathbf{x}+\lambda_{i, j} \mathbf{d}_{i}
\end{array}\right)^{\top}+\left(1-\sum_{i=1}^{M} \lambda_{i, j}^{-2}\right) Y\right] \in \operatorname{Feas}^{+}(\mathrm{QP}) .
$$

Finally, considering $j \rightarrow \infty$, we get $\lambda_{i, j}^{-1} \rightarrow 0, \lambda_{i, j}^{-2} \rightarrow 0$ and $\left(1-\sum_{i=1}^{M} \lambda_{i, j}^{-2}\right) \rightarrow 1$, which implies $\left[Y+\left(\begin{array}{cc}0 & \mathbf{0}^{\top} \\ \mathbf{0} & D\end{array}\right)\right] \in \operatorname{cl}\left(\mathrm{Feas}^{+}(\mathrm{QP})\right)$.

We now need the following lemma from [4].

Lemma 10 [4, Lemma 8] We have that the optimal value of $(\mathrm{QP})$ is equal to

$$
\inf \left\{\left\langle\left(\begin{array}{ll}
0 & \mathbf{c}^{\mathrm{T}} \\
\mathbf{c} & Q
\end{array}\right), Y\right\rangle \mid Y \in \operatorname{cl}\left(\mathrm{Feas}^{+}(\mathrm{QP})\right)\right\} .
$$

Using the obvious fact that the set of optimal solutions of an unbounded problem is empty and $\emptyset+\mathcal{X}=\emptyset$ for every set $\mathcal{X}$, we immediately get the following theorem from the previous two lemmas and the proof of Theorem 8 .

Theorem 11 Consider problems $(\mathrm{QP})$ and $\left(\mathrm{QP}_{\mathrm{C}}\right)$ such that Assumptions 1, 2 and 4 hold. Then $\operatorname{Val}(\mathrm{QP})=\operatorname{Val}\left(\mathrm{QP}_{\mathrm{C}}\right)$, and

$$
\mathrm{Opt}^{+}\left(\mathrm{QP}_{\mathrm{C}}\right)=\operatorname{conv}\left\{\left(\begin{array}{l}
1 \\
\mathbf{x}
\end{array}\right)\left(\begin{array}{l}
1 \\
\mathbf{x}
\end{array}\right)^{\top} \mid \mathbf{x} \in \mathrm{Opt}(\mathrm{QP})\right\}+\operatorname{conv}\left\{\left(\begin{array}{l}
0 \\
\mathbf{d}
\end{array}\right)\left(\begin{array}{l}
0 \\
\mathbf{d}
\end{array}\right)^{\top} \mid \begin{array}{l}
\mathbf{d} \in L_{\infty}, \\
\mathbf{d}^{\top} Q \mathbf{d}=0
\end{array}\right\} .
$$




\section{Main theorem}

We consider the following assumptions, which are simpler to verify and are more commonly used in the literature. Recall for these that $\mathcal{K} \subseteq \mathbb{R}^{n}$ is nonempty.

Assumption $5 \mathcal{K}$ is closed and $\mathcal{B}=\emptyset$.

Assumption $6 \mathcal{K}$ is closed and bounded.

Assumption $7 \mathcal{K}$ is closed and for all $\mathbf{d} \in \mathcal{K}_{\infty}, \mathbf{x} \in \mathcal{K}, \tilde{\lambda} \in \mathbb{R}_{+}$, there exists $\lambda \in \mathbb{R} \backslash(-\tilde{\lambda}, \tilde{\lambda})$ such that $\mathbf{x}+\lambda \mathbf{d} \in \mathcal{K}$.

Assumption $8 \mathcal{K}$ is closed and $\mathcal{K}_{\infty}=\operatorname{recc}(\mathcal{K})$, where

$$
\operatorname{recc}(\mathcal{K}):=\left\{\mathbf{d} \in \mathbb{R}^{n} \mid \mathbf{x}+\omega \mathbf{d} \in \mathcal{K} \quad \text { for all } \mathbf{x} \in \mathcal{K}, \omega \in \mathbb{R}_{+}\right\}
$$

(Note that, from the definition, we have $\mathcal{K}_{\infty} \supseteq \operatorname{recc}(\mathcal{K})$.)

Assumption $9 \mathcal{K}$ is closed and convex.

We now consider the following lemmas connected to these assumption.

Lemma 12 If Assumption 5 holds then Assumption 2 also holds.

Proof This is trivial to see.

Lemma 13 If Assumption 6 holds then Assumptions 2 and 4 also hold.

Proof If $\mathcal{K}$ is bounded then $\mathcal{K}_{\infty}=\{\mathbf{0}\}$ and the implication immediately follows.

Lemma 14 If Assumptions 1 and 7 hold then Assumptions 2 and 4 also hold.

Proof Suppose there exists $\mathbf{x} \in \mathcal{K}$ such that $\mathbf{a}_{i}^{\top} \mathbf{x}=b_{i}$ for all $i$. Then for all $\tilde{\lambda} \in \mathbb{R}_{+}$, $\mathbf{d} \in L_{\infty} \subseteq \mathcal{K}_{\infty}$, there exists $\lambda \in \mathbb{R} \backslash(-\tilde{\lambda}, \widetilde{\lambda})$ such that $\mathbf{x}+\lambda \mathbf{d} \in \mathcal{K}$. We then also have that $\mathbf{a}_{i}^{\top}(\mathbf{x}+\lambda \mathbf{d})=b_{i}$ for all $i$. Now combining this observation with Assumption 1 and $\mathcal{K}$ being closed, we get that Assumption 2 holds, from which we get that Assumption 4 also holds.

Lemma 15 If Assumption 8 holds then Assumption 7 also holds.

Proof Trivial from the definition of the recession cone.

Lemma 16 If Assumption 9 holds then 8 also holds.

Proof This is a well known result.

Combining these lemmas with Theorems 8 and 11 gives us the following theorem, which is the best approximation to the disproved Theorem 10 from [4].

Theorem 17 Consider problems $(\mathrm{QP})$ and $\left(\mathrm{QP}_{\mathrm{C}}\right)$ such that Assumption 1 holds and at least one of the following is true: 
1. Assumptions 2 and 3 hold,

2. Assumptions 2 and 4 hold,

3. Assumptions 3 and 5 hold,

4. Assumptions 4 and 5 hold,

5. Assumption 6 holds,

6. Assumption 7 holds,

7. Assumption 8 holds,

8. Assumption 9 holds.

Then $\operatorname{Val}(\mathrm{QP})=\operatorname{Val}\left(\mathrm{QP}_{\mathrm{C}}\right)$ and we have that

$$
\mathrm{Opt}^{+}\left(\mathrm{QP}_{\mathrm{C}}\right)=\operatorname{conv}\left\{\left(\begin{array}{l}
1 \\
\mathbf{x}
\end{array}\right)\left(\begin{array}{l}
1 \\
\mathbf{x}
\end{array}\right)^{\top} \mid \mathbf{x} \in \mathrm{Opt}(\mathrm{QP})\right\}+\operatorname{conv}\left\{\left(\begin{array}{l}
0 \\
\mathbf{d}
\end{array}\right)\left(\begin{array}{l}
0 \\
\mathbf{d}
\end{array}\right)^{\top} \mid \begin{array}{l}
\mathbf{d} \in L_{\infty}, \\
\mathbf{d}^{\top} Q \mathbf{d}=0
\end{array}\right\} .
$$

Note from this theorem that, in such cases as described, $\mathrm{Opt}(\mathrm{QP})=\emptyset$ if and only if Opt $\left(\mathrm{QP}_{\mathrm{C}}\right)=\emptyset$. In other words, the optimal solution in $(\mathrm{QP})$ is attained if and only if the optimal solution to $\left(\mathrm{QP}_{\mathrm{C}}\right)$ is attained.

\section{Dealing with one quadratic inequality constraint}

In this section we show that Corollary 14 from [4], stating that Theorem 10 from the same paper is valid for $\mathcal{K}$ defined by a (possibly non-convex) quadratic inequality, is still true even though Theorem 10 is disproved. Recall that we consider $\mathcal{K}=\left\{\mathbf{x} \in \mathbb{R}^{n} \mid \mathbf{x}^{\top} P \mathbf{x}+2 \mathbf{p}^{\top} \mathbf{x}+p_{0} \leq 0\right\}$ with $P \in \mathcal{S}^{n}, \mathbf{p} \in \mathbb{R}^{n}$ and $p_{0} \in \mathbb{R}$, such that $\mathcal{K} \neq \emptyset$.

First we provide an explicit description of $\mathcal{K}_{\infty}$.

Theorem 18 For $\mathcal{K}=\left\{\mathbf{x} \in \mathbb{R}^{n} \mid \mathbf{x}^{\top} P \mathbf{x}+2 \mathbf{p}^{\top} \mathbf{x}+p_{0} \leq 0\right\}$ we have

1. $\mathcal{K}_{\infty} \subseteq\left\{\mathbf{d} \in \mathbb{R}^{n} \mid \mathbf{d}^{\top} P \mathbf{d} \leq 0\right\}$,

2. If $P \notin \mathcal{S}_{+}$then $\mathcal{K}_{\infty}=\left\{\mathbf{d} \in \mathbb{R}^{n} \mid \mathbf{d}^{\top} P \mathbf{d} \leq 0\right\}=\operatorname{cl}\left\{\mathbf{d} \in \mathbb{R}^{n} \mid \mathbf{d}^{\top} P \mathbf{d}<0\right\}$,

3. If $P \in \mathcal{S}_{+}$and $\mathcal{K} \neq \emptyset$, then $\mathcal{K}_{\infty}=\operatorname{recc}(\mathcal{K})=\left\{\mathbf{d} \in \mathbb{R}^{n} \mid \mathbf{d}^{\top} P \mathbf{d}=0, \mathbf{p}^{\top} \mathbf{d} \leq 0\right\}$.

Proof We shall prove each point in turn.

1. We consider an arbitrary $\mathbf{d} \in \mathcal{K}_{\infty}$. There exists a sequence $\left\{\left(\lambda_{i}, \mathbf{x}_{i}\right) \mid i \in \mathbb{N}\right\} \subseteq \mathbb{R}_{+} \times \mathcal{K}$ such that $\lim _{i \rightarrow \infty} \lambda_{i}=0$ and $\lim _{i \rightarrow \infty} \lambda_{i} \mathbf{x}_{i}=\mathbf{d}$. We then have that

$$
\begin{aligned}
0 & \geq \lim _{i \rightarrow \infty}\left(\lambda_{i}^{2}\left(\mathbf{x}_{i}^{\top} P \mathbf{x}_{i}+2 \mathbf{p}^{\top} \mathbf{x}_{i}+p_{0}\right)\right) \\
& \left.=\lim _{i \rightarrow \infty}\left(\left(\lambda_{i} \mathbf{x}_{i}\right)^{\top} P\left(\lambda_{i} \mathbf{x}_{i}\right)+2 \lambda_{i} \mathbf{p}^{\top}\left(\lambda_{i} \mathbf{x}_{i}\right)+\lambda_{i}^{2} p_{0}\right)\right) \\
& =\mathbf{d}^{\top} P \mathbf{d} .
\end{aligned}
$$

2. We consider an arbitrary $\mathbf{d} \in \mathbb{R}^{n}$ such that $\mathbf{d}^{\top} P \mathbf{d}<0$. There exists a $\hat{\omega}>0$ such that for all $\omega \geq \hat{\omega}$ we have $\omega^{2} \mathbf{d}^{\top} P \mathbf{d}+2 \omega \mathbf{p}^{\top} \mathbf{d}+p_{0} \leq 0$. We now define the sequence $\left\{\left(\lambda_{i}, \mathbf{x}_{i}\right) \mid i \in \mathbb{N}\right\} \subseteq \mathbb{R}_{+} \times \mathcal{K}$ such that $\lambda_{i}=(\hat{\omega}+i)^{-1}$ and 
$\mathbf{x}_{i}=(\hat{\omega}+i) \mathbf{d}$. Using this sequence, we can immediately see that $\mathbf{d} \in \mathcal{K}_{\infty}$, and thus $\mathcal{K}_{\infty} \supseteq\left\{\mathbf{d} \in \mathbb{R}^{n} \mid \mathbf{d}^{\top} P \mathbf{d}<0\right\}$.

We now suppose that $P \notin \mathcal{S}_{+}$and consider an arbitrary $\mathbf{x} \in \mathbb{R}^{n}$ such that $\mathbf{x}^{\top} P \mathbf{x}=0$. There exists $\mathbf{y}$ such that $\mathbf{y}^{\top} P \mathbf{y}<0$. As $(-\mathbf{y})^{\top} P(-\mathbf{y})=\mathbf{y}^{\top} P \mathbf{y}<0$, we may assume w.l.o.g. that $\mathbf{x}^{\top} Q \mathbf{y} \leq 0$. Then for all $\varepsilon>0$ we have $(\mathbf{x}+\varepsilon \mathbf{y})^{\top} P(\mathbf{x}+\varepsilon \mathbf{y})<0$. Therefore, by the previous paragraph, $\mathbf{x}+\varepsilon \mathbf{y} \in \mathcal{K}_{\infty}$ for all $\varepsilon>0$. Hence, using the fact that $\mathcal{K}_{\infty}$ is a closed cone, we have $\mathbf{x} \in \operatorname{cl}\left\{\mathbf{d} \in \mathbb{R}^{n} \mid \mathbf{d}^{\top} P \mathbf{d}<0\right\} \subseteq \operatorname{cl} \mathcal{K}_{\infty}=\mathcal{K}_{\infty}$.

3. From now on we suppose that $P \in \mathcal{S}_{+}$and $\mathcal{K} \neq \varnothing$.

We first consider an arbitrary $\mathbf{d} \in \mathcal{K}_{\infty}$. From the first part, and $P \in \mathcal{S}_{+}$, we have $\mathbf{d}^{\top} P \mathbf{d}=0$, and there exists a sequence $\left\{\left(\lambda_{i}, \mathbf{x}_{i}\right) \mid i \in \mathbb{N}\right\} \subseteq \mathbb{R}_{+} \times \mathcal{K}$ such that $\lim _{i \rightarrow \infty} \lambda_{i}=0$ and $\lim _{i \rightarrow \infty} \lambda_{i} \mathbf{x}_{i}=\mathbf{d}$. We then have that

$$
\begin{aligned}
2 \mathbf{p}^{\top} \mathbf{d} & =\lim _{i \rightarrow \infty}\left(\lambda_{i} 2 \mathbf{p}^{\top} \mathbf{x}_{i}\right) \\
& \leq \lim _{i \rightarrow \infty}\left(\lambda_{i}\left(-\mathbf{x}_{i}^{\top} P \mathbf{x}_{i}-p_{0}\right)\right) \\
& \leq \lim _{i \rightarrow \infty}\left(-\lambda_{i} p_{0}\right) \\
& =0 .
\end{aligned}
$$

Therefore $\mathcal{K}_{\infty} \subseteq\left\{\mathbf{d} \in \mathbb{R}^{n} \mid \mathbf{d}^{\top} P \mathbf{d}=0, \mathbf{p}^{\top} \mathbf{d} \leq 0\right\}$.

We next consider an arbitrary $\mathbf{d} \in \mathbb{R}^{n}$ such that $\mathbf{d}^{\top} P \mathbf{d}=0$ and $\mathbf{p}^{\top} \mathbf{d} \leq 0$. As $P \in \mathcal{S}_{+}$, we have $P \mathbf{d}=\mathbf{0}$. Now, for any $\mathbf{x} \in \mathcal{K}$ and $\omega \geq 0$, we observe that

$$
\begin{aligned}
(\mathbf{x}+\omega \mathbf{d})^{\top} & P(\mathbf{x}+\omega \mathbf{d})+2 \mathbf{p}^{\top}(\mathbf{x}+\omega \mathbf{d})+p_{0} \\
& =\underbrace{\mathbf{x}^{\top} P \mathbf{x}+2 \mathbf{p}^{\top} \mathbf{x}+p_{0}}_{\leq 0}+\underbrace{2 \omega \mathbf{p}^{\top} \mathbf{d}}_{\leq 0} \leq 0,
\end{aligned}
$$

and thus $\mathbf{x}+\omega \mathbf{d} \in \mathcal{K}$, from which it can immediately be seen that $\mathbf{d} \in \operatorname{recc}(\mathcal{K}) \subseteq \mathcal{K}_{\infty}$

We now get the following theorem connecting the quadratic case to one of our earlier assumptions.

Theorem 19 For $\mathcal{K}$ as given at the start of this section, we have that Assumption 7 holds.

Proof We consider an arbitrary $\mathbf{d} \in \mathcal{K}_{\infty}, \mathbf{x} \in \mathcal{K}, \tilde{\lambda} \in \mathbb{R}_{+}$, and let $\lambda \in \mathbb{R} \backslash(-\tilde{\lambda}, \tilde{\lambda})$ such that

$$
\lambda= \begin{cases}-\tilde{\lambda} & \text { if } \mathbf{d}^{\top}(2 P \mathbf{x}+2 \mathbf{p}) \geq 0 \\ +\widetilde{\lambda} & \text { if } \mathbf{d}^{\top}(2 P \mathbf{x}+2 \mathbf{p})<0\end{cases}
$$


From the previous theorem we have that $\mathbf{d}^{\top} P \mathbf{d} \leq 0$, and thus

$$
\begin{aligned}
(\mathbf{x}+\lambda \mathbf{d})^{\top} & P(\mathbf{x}+\lambda \mathbf{d})+2 \mathbf{p}^{\top}(\mathbf{x}+\lambda \mathbf{d})+p_{0} \\
= & \underbrace{\mathbf{x}^{\top} P \mathbf{x}+2 \mathbf{p}^{\top} \mathbf{x}+p_{0}}_{\leq 0}+\underbrace{\lambda \mathbf{d}^{\top}(2 P \mathbf{x}+2 \mathbf{p})}_{\leq 0}+\underbrace{\lambda^{2} \mathbf{d}^{\top} P \mathbf{d}}_{\leq 0} \leq 0 .
\end{aligned}
$$

Therefore $\mathbf{x}+\lambda \mathbf{d} \in \mathcal{K}$, and so Assumption 7 holds.

Corollary 20 Corollary 14 from [4] is correct.

\section{Conclusion}

Due to precise observation of P.J.C. Dickinson, it has been found that the proof of Lemma 9 in [4] by Eichfelder and Povh has a nontrivial gap. All three authors of this erratum paper worked jointly to provide new proofs to show that although Theorem 10 from [4] is not true in general, it is possible to correct this by adding some commonly used assumptions, as presented in Theorem 17. Using this, the three authors of this erratum were then able to show that Corollary 14 from [4] is fortunately still correct.

Acknowledgments This work was done while P.J.C. Dickinson was working at the Johann Bernoulli Institute for Mathematics and Computer Science, University of Groningen, The Netherlands, and we would like to thank the support that they provided. The third author wishes to thank to Slovenian research agency for support via program P1-0383 and project L74119 and to Creative Core FISNM-3330-13-500033.

\section{References}

1. Auslender, A., Teboulle, M.: Asymptotic cones and functions in optimization and variational inequalities. In: Springer Monographs in Mathematics. Springer, New York (2003)

2. Bomze, I., Jarre, F.: A note on Burer's copositive representation of mixed-binary QPs. Optim. Lett. 4(3), 465-472 (2010)

3. Burer, S.: On the copositive representation of binary and continuous nonconvex quadratic programs. Math. Program. 120(2), 479-495 (2009)

4. Eichfelder, G., Povh, J.: On the set-semidefinite representation of nonconvex quadratic programs over arbitrary feasible sets. Optim. Lett. doi:10.1007/s11590-012-0450-3 (2012) 